\title{
Escritura expositiva en tres niveles de escolaridad: coherencia y dominio de recursos lingüísticos
}

\author{
Soledad Concha* \\ Soledad Aravena** \\ Carmen Julia Coloma ${ }^{* * *}$ \\ Verónica Romero****
}

\begin{abstract}
Resumen
El estudio explora la capacidad de estudiantes de tres niveles de escolaridad $-5^{\circ}$ Básico, $1^{\circ}$ Medio y $4^{\circ}$ Medio- para producir textos expositivos coherentes utilizando los recursos lingüísticos con que se construye la coherencia local en la escritura expositiva. Se trabajó con alumnos de dos colegios municipalizados de Santiago, quienes redactaron textos expositivos a partir de un ítem de respuesta abierta extensa. Sus textos fueron codificados con dos rúbricas para medir la coherencia local (CL) y los recursos lingüísticos involucrados en la coherencia local (RCL). Los resultados muestran un ascenso significativo en el desempeño para la medida RCL a lo largo de los tres niveles escolares, pero no para la medida CL.
\end{abstract}

Palabras clave: Producción escrita, texto expositivo, coherencia, recursos lingüísticos

\begin{abstract}
The study focused on 5th grade, 9th grade and 12th grade students' ability to produce coherent expository texts and to use the linguistic resources with which local coherence is achieved in expository writing. Participants belonged to two public schools in Santiago, Chile, and their expository writing was elicited with writing prompts. Products of writing were coded using two different rubrics: Local Coherence (LC) and Local Coherence Resources (LCR). Results reveal a significant improvement for the measure LCR across age groups, but not for LC.
\end{abstract}

Key words: Writing, expository text, coherence, linguistic resources

* Doctora en Educación. Académica de la Facultad de Educación de la Universidad Diego Portales.Soledad.Concha@udp.cl Fono:6768503

** Magister en Lingüística. Académica de la Facultad de Filosofía y Humanidades de la Universidad Alberto Hurtado, y de la Facultad de Letras de la Pontificia Universidad Católica de Chile, Solaravena@gmail.com Fono:6920242

*** Magíster en Lingüística, Académica de la Escuela de Fonoaudiología de la Universidad de Chile,ccoloma@med.uchile.cl

***** Magíster en Literatura, Académica de la Facultad de Ciencias Sociales e Historia, Universidad Diego Portales, veronica.romero@udp.cl, F. 6768503 


\section{Introducción}

A diferencia de la conversación o de la narración, la exposición no forma parte del repertorio comunicativo cotidiano y familiar de los niños. La exposición es una práctica discursiva que se adquiere y desarrolla durante la escolarización, con el objetivo de llegar a convertirse en uno de los pilares fundamentales de transmisión del conocimiento en las diversas materias escolares y, posteriormente, en muchas áreas de la enseñanza superior, tanto técnica y profesional como científica. Las características del texto expositivo -su contenido proposicional y su estructura lógica compleja- sumadas al potencial epistémico de la escritura -como recurso de organización del pensamiento y de generación de conocimiento-, hacen que el logro de la escritura expositiva sea un objetivo central de los años escolares terminales. Así, en la llamada sociedad del conocimiento, aprender a escribir textos expositivos significa, en último término, tener la posibilidad de adquirir una herramienta efectiva tanto para el acceso a la información, como para la construcción de nuevos saberes.

Una de las características centrales de los discursos expositivos es que requieren del manejo de un repertorio de dominios lingüístico-discursivos que funcionan como elementos de organización y expresión, y que se identifican con tradiciones culturales como la academia y con diversos contextos laborales. Silvestri (2002) propone que la adquisición discursivo-textual que implica la habilidad para comprender y producir textos -orales o escritos- de diversos tipos y géneros depende de las oportunidades de interacción cultural que experimenten los sujetos. En efecto, a diferencia de la adquisición temprana del léxico y la gramática de la propia lengua, que ocurre durante los primeros 6 años de vida, la capacidad para producir y comprender discursos académicos se produce en la medida en que los sujetos tienen oportunidades de participar en situaciones en que puedan adquirir determinadas formas del lenguaje y la manera de operar sobre ellas según códigos culturales determinados. Al respecto, Jacobs (1990) propone que la escritura expositiva forma parte de las convenciones de una cultura académica que impone a los estudiantes el desafío de aprender a construir la realidad según las formas que se valoran en los contextos escolares. El tipo de razonamiento de la prosa expositiva se traspasa por generaciones en culturas en las que la escolarización es común y tiene un efecto en la comunicación oral y en los hábitos de pensamiento usados y valorados en dichas comunidades: el razonamiento lógico, la argumentación, son patrones de pensamiento que derivan de las convenciones de la prosa académica. Un uso experto de dichos tipos de razonamiento u organización de las ideas se asocia con discursos en los que los conocimientos han sido transformados según estructuras de organización jerárquica que requieren de los recursos lingüístico-discursivos propios de la escritura académica (por ejemplo, relaciones lógicas y conectores).

Durst (1987) describe la producción escrita académica como una actividad en la que los escritores ejercitan sus habilidades de razonamiento crítico. Para él, este tipo de escritura involucra reflexionar respecto de las relaciones que existen 
entre las ideas y los eventos sobre los cuales se escribe (347), a diferencia de lo que ocurre con otros textos como las narraciones. El resultado de este tipo de procesamiento se expresa en el texto en relaciones como la causalidad y la adversatividad en lugar de la temporalidad y la adición.

En términos cognitivos, construir un texto bien formado requiere haber adquirido la representación mental apropiada de un tipo particular de discurso. Para ello, los niños deben integrar los componentes estructurales básicos en una pieza discursiva unificada. En el texto narrativo, por ejemplo, el componente estructural básico está dado por los eventos. Por tanto un texto narrativo bien construido requiere de un esquema integrador de los eventos o acciones. En cambio, el texto expositivo está orientado por el tópico. El tópico del discurso funciona como una categoría supraordinada bajo la cual el resto de la información debe organizarse. En este sentido, la construcción del texto expositivo implica establecer y relacionar conceptos y categorías para formar un sistema subordinado al tema central del discurso (Berman y Nir-Sagiv, 2007).

No obstante la valoración cultural de este tipo de patrones, es común que sujetos de distintas edades presenten dificultades para imponer un esquema académico a sus discursos. Dichas dificultades se han investigado desde diferentes perspectivas y entregan luces sobre el desarrollo de estos dominios. Desde una perspectiva de adquisición, por ejemplo, es interesante constatar en estudios realizados con estudiantes angloparlantes que los niños son capaces de establecer relaciones lógicas como la causalidad en el discurso oral (Clark, 2003) antes de aprender a escribir; no obstante, este conocimiento no parece transferir naturalmente a la escritura. En efecto, las ideas en los textos de escritores inexpertos tienden a coordinarse mediante la yuxtaposición o la adición (Bereiter y Scardamalia, 1987), tal como sucedió en las primeras etapas de la adquisición del discurso oral. En este sentido, la evidencia estaría sugiriendo un desfase en la capacidad para expresar ideas más complejas y coordinar informaciones entre el medio oral y el escrito. Vale la pena recalcar del trabajo de Bereiter y Scardamalia (1987) que la evolución en este sentido involucra un procesamiento cognitivo cada vez más complejo que permita no solo mantener en línea dos ideas, sino además una relación lógica que las asocie; así, los escritores más sofisticados serían capaces de procesar varias ideas y relaciones lógicas a la vez, de tal manera que no solo "dicen" el conocimiento cuando escriben, sino que lo "transforman" en nuevas estructuras de información.

Dichos estudios permiten trazar cierto camino evolutivo respecto de la capacidad para coordinar ideas por escrito (coherencia local) al menos en los primeros años de escolaridad. Por ejemplo, Favart y Passerault (1996) observaron este dominio en las narrativas producidas por estudiantes de $2^{\circ}, 3^{\circ}, 4^{\circ}$ y $5^{\circ}$ Básico y concluyeron que se incrementaba significativamente, entre los niveles, la cantidad de conectores producidos a nivel global y, muy especialmente, a nivel local. Como señalaran Peterson y McCabe (1987), en los niños menores tiende a predominar el uso polifuncional del conector ' $y$ ' (puede expresar adición, causalidad, adversatividad, etc.). De este modo, la evolución no solo se 
relaciona con el número de conectores usados sino con la creciente especificación en el tipo de relaciones entre ideas del texto. Una cuestión importante en estos estudios es la constatación de que los escolares del primer ciclo tienden a utilizar relaciones menos complejas semánticamente, como la temporalidad, mientras que la causalidad y las relaciones de propósito aparecen con menos frecuencia en ellos.

Con respecto a los estudios disponibles con escolares de Enseñanza Media (EM), los resultados no son concluyentes respecto a la secuencia evolutiva de la producción escrita. Estudios como los de Langer (1986) y Scardamalia y Paris (1985), señalan los 14 años como una edad clave en el desarrollo de los saberes lingüístico-discursivos asociados a la escritura académica. Por una parte, Langer (1986) afirma que a esa edad los sujetos comienzan a usar más frecuentemente estructuras jerárquicas inferiores (predicados retóricos) como las de causalidad, alternativa, evidencia y adversatividad, lo que produciría discursos más complejos y más coherentes. Sin embargo, no existe unanimidad entre las investigaciones acerca de la etapa precisa en que se adquieren estas competencias. Algunos estudios indican que el manejo de relaciones de coherencia y conectores argumentativos aparece entre los 10 y 14 años, mientras que otros señalan que este dominio no se ha alcanzado del todo a los 14 años y que solo a los 16 años es posible encontrar textos argumentativos con una estructura jerárquica guiada por propósitos semánticos claros (una revisión puede encontrarse en Piolat, Roussey y Gombert, 1999).

De nuestro conocimiento, en Chile no se han difundido estudios que analicen la escritura expositiva de los estudiantes del sistema escolar desde un punto de vista evolutivo. Sin embargo, se cuenta con trabajos que confirman el dominio precario de la producción escrita en los estudiantes del país en distintos niveles y respecto de dimensiones también diversas de la escritura. En particular, una serie de investigaciones realizadas en la V Región en niveles terminales de EB y también en EM, sugieren en nuestros escolares un conocimiento impreciso de los requisitos de la comunicación escrita, por lo que enfrentan la tarea de escritura con escaso manejo de recursos lingüísticos y estrategias adecuadas.

$\mathrm{Al}$ respecto, se ha encontrado que los estudiantes de $8^{\circ}$ Básico conciben el proceso de escritura en un esquema lineal que deja fuera conductas de planificación y revisión, y que tienden a valorar cuestiones poco ligadas a la comunicación eficiente, como la ortografía y la presentación (Marinkovich y Poblete, 2000). También se ha detectado en $1^{\circ}$ y $2^{\circ}$ Medio un acercamiento intuitivo a la tarea de escritura y ausencia de conocimientos específicos respecto de la complejidad de la comunicación escrita (Crespo, 1998). En cuanto al desempeño específico en determinadas etapas de la escolaridad, hay evidencia de que los estudiantes de $8^{\circ}$ Básico, enfrentados a la tarea de escribir un texto argumentativo, demuestran un dominio escaso de los componentes macro y superestructurales del discurso, mientras que su rendimiento es relativamente bueno en el logro de relaciones de coherencia local. Entre estas relaciones, las correferencias nominales y verbales son las menos afectadas, en tanto que las 
relaciones causa-efecto son las que presentan más dificultades (Parodi, 2000). Este dato resulta interesante en la medida en que este tipo de relaciones y otras de mayor complejidad, como las de contraargumentación, por ejemplo, son las relaciones lógicas propias de la estructura expositiva y de los textos académicos en general.

Se ha encontrado además que los estudiantes de $3^{\circ}$ medio mejoran su desempeño en escritura en el corto plazo después de una intervención destinada a incrementar la reflexión y el conocimiento sobre estrategias de producción escrita. Sin embargo, se ha demostrado que estos aprendizajes no se sostienen en el tiempo sin una práctica sistemática y además no son transferibles a otras materias (Velásquez y Alonzo, 2007). Este tipo de hallazgos son especialmente reveladores para el presente estudio, que junto con describir el dominio de los recursos de la coherencia local en distintos niveles de la escolaridad, pretende contribuir a la reflexión sobre la didáctica de estas habilidades en el aula.

Por último, en un estudio previo (Concha, 2007) se exploró el dominio de la coherencia local y de los recursos de la lengua asociados a la coherencia local, en muestras de escritura argumentativa extraídas de 100 estudiantes de $4^{\circ}$ Medio de liceos municipalizados de Santiago. Los resultados obtenidos señalan que, en promedio, el desempeño de los estudiantes es mejor en coherencia local que en el uso de recursos de la coherencia local. En efecto, los estudiantes produjeron en promedio textos con algunas secuencias incoherentes, pero que no impedían al lector extraer la idea global del texto. No obstante, los señalados textos obtenían coherencia por vía de relaciones lógicas, conectores y mecanismos para recuperar información que son más propios de la oralidad (por ejemplo, adición, temporalidad) y que no implicaban mayor interrelación entre los contenidos. Se concluye del trabajo que los estudiantes de la muestra no reflejan dominio de los mecanismos de la lengua convencionales al tipo de discurso académico y más bien logran la coherencia aplicando recursos que transfieren de la comunicación oral. Adicionalmente, en este estudio se asocia este comportamiento con una falta de experiencias de didáctica específica de la coherencia local y sus recursos.

Junto a los hallazgos de la investigación, se cuenta también con una serie de indicios que sugieren problemas de desempeño en la producción escrita expositiva de los estudiantes de nuestro país, no solo al término de la Enseñanza Básica, sino también al final de la Enseñanza Media e incluso en la Educación Superior. En cuanto a los primeros niveles, en Chile se cuenta con los comentarios en los reportes oficiales del Sistema de Medición de la Calidad de la Educación (SIMCE) referidos a las respuestas de desarrollo. Estos informes resultan particularmente importantes para el presente estudio, puesto que señalan una debilidad mayor en la comunicación escrita no narrativa, eje central del currículum vigente de EM (MINEDUC, Marco Curricular 1998/2005). Respecto de los niveles superiores, es sabido que actualmente las universidades chilenas deben implementar reforzamientos tras diagnosticar el bajo desempeño en producción textual que muestran los estudiantes de primer año. En esta línea, se han documentado 
interesantes trabajos que apuntan a la necesidad urgente de desarrollar procedimientos más eficaces de alfabetización académica (Carlino, 2008; Pereira y Di Stéfano, 2007; Velásquez, 2005; Villalón y Mateos, 2009).

Frente a este problema y en respuesta a la ausencia de explicaciones evolutivas de estos fenómenos en nuestro país, la presente investigación se propone contribuir a la comprensión de la configuración de la coherencia local de textos expositivos producidos por estudiantes chilenos de diferentes niveles, con miras a hacer aportes que enriquezcan, en alguna medida, la didáctica de estas habilidades. En particular, se abordan los siguientes objetivos:

1. Describir el desempeño de los estudiantes de $5^{\circ}$ Básico $\left(5^{\circ} \mathrm{B}\right), 1^{\circ} \mathrm{Me}$ dio $\left(1^{\circ} \mathrm{M}\right)$ y $4^{\circ}$ Medio $\left(4^{\circ} \mathrm{M}\right)$ en la producción de textos expositivos coherentes.

2. Describir el desempeño de los estudiantes de $5^{\circ} \mathrm{B}, 1^{\circ} \mathrm{M}$ y $4^{\circ} \mathrm{M}$ en el uso de los recursos lingüísticos involucrados en la coherencia local (RCL) en la escritura expositiva.

3. Determinar si existen diferencias en los desempeños de estudiantes de cada uno de los tres niveles, en la coherencia local (CL) de sus textos expositivos.

4. Determinar si existen diferencias en los desempeños de estudiantes de cada uno de los tres niveles en la utilización de RCL en la coherencia local en la escritura expositiva.

\section{Metodología}

\section{Selección de establecimientos y participantes}

Se trabajó con una muestra de 149 estudiantes (tabla I) de dos establecimientos de la comuna de Lo Barnechea de dependencia municipalizada (E1 y E2, en los datos que se muestran a continuación). Se escogieron establecimientos municipalizados por su alta representatividad en el sistema escolar nacional. Ambos establecimientos presentan puntajes promedio de SIMCE 2007 bajo la media nacional (en torno a los 20 puntos), los que son característicos de su tipo de dependencia y nivel socioeconómico. Se optó por dos escuelas de nivel socioeconómico medio-bajo en la escala establecida por el Ministerio de Educación, porque se trata de un grupo socioeconómico de alta frecuencia en el tipo de dependencia municipalizada (MINEDUC 2008).

En una primera evaluación, participaron los 221 estudiantes que conformaban la totalidad de inscritos en los niveles y colegios seleccionados con el objetivo de registrar las conductas y desempeños típicos de cada nivel. Por esta razón, los alumnos integrados, que evidencian desempeños que se alejan ostensiblemente de la media, no fueron considerados en la muestra (aunque rindieron las pruebas). De los 221 sujetos que participaron en la primera evaluación, se 
seleccionó una muestra de 149 estudiantes a partir del análisis de la validez de los instrumentos que respondieron y no de la calidad de sus respuestas. Lo anterior se explica más adelante en la descripción de la tarea. Como puede verse en la tabla 1, la distribución de estudiantes no es homogénea en los tres niveles escolares analizados y disminuye notablemente entre $5^{\circ} \mathrm{B}$ y $4^{\circ} \mathrm{M}$, debido al ausentismo y la deserción escolar que evidencian estos establecimientos y no a decisiones muestrales.

Tabla 1: Composición de la muestra

\begin{tabular}{|c|c|c|c|}
\hline Nivel & E1 & E2 & Total \\
\hline $5^{\circ}$ Básico & 53 & 20 & 73 \\
\hline $1^{\circ}$ Medio & 32 & 22 & 54 \\
\hline $4^{\circ}$ Medio & 12 & 10 & 22 \\
\hline Total & 97 & 52 & 149 \\
\hline
\end{tabular}

\section{Elaboración y validación de instrumentos}

Se elaboraron tres ítemes (enunciados con instrucciones para la escritura) de respuesta abierta extensa para cada nivel escolar ( 9 en total) con el fin de motivar la escritura de un texto expositivo. Se optó por enunciados breves (dos oraciones), redactados con vocabulario frecuente, que no demandaran dificultad en la comprensión lectora. Los temas de los enunciados fueron seleccionados por la vía de una encuesta de intereses y conocimientos aplicada previamente a una muestra de estudiantes comparables. El objetivo fue evitar que los estudiantes pudieran redactar textos incoherentes a causa de la falta de interés o conocimiento previo de los temas propuestos (Kellog, 1987; Hayes, 2000). La totalidad de los ítemes fue validada mediante juicio de expertos. Cuatro expertos evaluaron su calidad considerando: la edad de los niños, su curso y la potencialidad del ítem para motivar la escritura. Para tales efectos, recibieron electrónicamente una copia de los instrumentos y una pauta de evaluación con los criterios señalados. Sus comentarios permitieron modificar y, en algunos casos, reformularlos completamente antes de ser aplicados.

Otro procedimiento de validación consistió en el análisis del funcionamiento de los ítemes. Para ello, los estudiantes de cada nivel fueron divididos en tres grupos equivalentes en número. Así, de la muestra original de 221 estudiantes, en $5^{\circ} \mathrm{B} 36$ estudiantes respondieron el ítem 1, 36 el ítem 2 y 37 el ítem 3; de $1^{\circ} \mathrm{M}, 25$ escolares respondieron el ítem 1,25 el ítem 2 y 27 el ítem $3 ;$ de $4^{\circ} \mathrm{M}, 11$ alumnos respondieron el ítem 1, 11 el ítem 2 y 13 el ítem 3. Con las respuestas obtenidas se realizó un análisis, según los siguientes criterios: porcentaje de abstención de respuesta, extensión de las respuestas obtenidas, variedad de contenidos y formas de iniciar las respuestas. De los resultados de este procedimiento se eliminó un ítem por nivel, lo que implicó que, para el análisis de datos, se consideraran finalmente 2 ítemes por nivel. Lo anterior significó que la muestra original se redujo a 149 sujetos con sus respectivas respuestas (una respuesta por participante): 73 de $5^{\circ} \mathrm{B}, 54$ de $1^{\circ} \mathrm{M}$ y 22 de $4^{\circ} \mathrm{M}$. 


\section{Procedimientos de recolección de datos}

La aplicación de los ítemes se realizó en un día de clases y los cursos trabajaron en su sala habitual con la presencia de un asistente de investigación y el profesor de lenguaje. El asistente de investigación entregó instrucciones generales, evitando en todo momento dar información que pudiera influir en las respuestas de los estudiantes. Para asegurar la homogeneidad del procedimiento, los asistentes debieron aprender un guión con las instrucciones y recibieron recomendaciones para responder posibles preguntas de los alumnos. Por su parte, el o la profesora no participó de este proceso sino que su presencia tenía por objeto resguardar la disciplina del curso.

\section{Procedimientos de análisis de datos}

Las 149 muestras de escritura recogidas se codificaron mediante dos rúbricas, CL y RCL, que fueron elaboradas y validadas en un estudio anterior (Concha, 2007) y que se muestran en las tablas 2 y 3 , a continuación. Ambas rúbricas constan de cuatro niveles, que se gradúan desde la ausencia del criterio analizado hasta el dominio del mismo. A cada nivel se le asigna un puntaje de 1 a 4 . La medida CL establece cuatro niveles que se determinan por la presencia de problemas de coherencia local y su influencia en la facilidad con la que el lector puede construir el significado global del texto. Se basa en la propuesta de Wikborg (1990) de evaluar la coherencia focalizándose en las interrupciones que debe realizar el lector durante el proceso de comprensión del texto. Se asume que la coherencia no es un fenómeno de los textos sino un fenómeno cognitivo que involucra una compleja interacción de principios semánticos, pragmáticos y lógicos que tanto el escritor como el lector activan para construir el significado del texto (Charolles, 1983; Lee, 2002; Hobbs, 1983).

Tabla 2: Rúbrica de Coherencia Local (CL)

\begin{tabular}{|l|l|l|l|}
\hline \multicolumn{1}{|c|}{1} & \multicolumn{1}{|c|}{2} & \multicolumn{1}{c|}{3} & \multicolumn{1}{c|}{4} \\
\hline El estudiante & El estudiante & El estudiante & El estudiante \\
produce un & produce un texto & produce un & produce un texto \\
texto en el que & en el que, si & texto que, si & que no presenta \\
predominan & bien hay algunas & bien presenta & problemas de \\
los errores de & secuencias & algunas secuencias & coherencia local \\
coherencia local, & incoherentes, en & lo que permite \\
lo que impide al & cantidad de errores & general permite & que el lector \\
lector seguir la & de coherencia & al lector seguir la & siga fluidamente \\
lectura fluidamente & hace difícil para & lectura fluidamente & la lectura y \\
y determinar el & el lector seguir la & y determinar el & que determine \\
sentido global del & lectura fluidamente & sentido global del & fácilmente el \\
texto. & y determinar el & texto. & sentido global del \\
texto.
\end{tabular}


La medida RCL, en tanto, clasifica las muestras de escritura según el dominio que demuestran los escritores de aquellos recursos lingüísticos que favorecen la producción de textos expositivos coherentes. Dichos recursos lingüísticos son: conectores, relaciones lógicas (por ejemplo, adversatividad, reformulación) y mecanismos de referencialidad (por ejemplo pronominalización, correferencialidad y anáforas).

Esta rúbrica se basa en la propuesta de Lee (2002) respecto de que el escritor cuenta con una serie de recursos lingüísticos que le permitirían lograr la coherencia en los distintos niveles en los que se articula el significado (por ejemplo, en el plano de la cohesión, de la progresión temática y de la consideración de las necesidades informativas del lector). Se consideran también una serie de trabajos que caracterizan los mecanismos de la coherencia local que son convencionales a la escritura académica (tales como relaciones de adversatividad o condicionalidad con y sin conectores), en oposición a aquellos más comunes de la oralidad o la narrativa (por ejemplo: conector 'y' polifuncional, relaciones de adición, temporalidad). El desarrollo de ellos se da a lo largo de la escolaridad (Durst, 1987; Langer, 1986; McCutchen y Perfetti, 1983: Borzone y Diuk, 2003).

Tabla 3: Rúbrica de Recursos de la Coherencia Local (RCL)

\begin{tabular}{|c|c|c|c|}
\hline 1 & 2 & 3 & 4 \\
\hline $\begin{array}{l}\text { El texto } \\
\text { demuestra una } \\
\text { incapacidad } \\
\text { para utilizar los } \\
\text { recursos de la } \\
\text { coherencia local. } \\
\text { Por ejemplo, la } \\
\text { mayoría de las } \\
\text { relaciones lógicas, } \\
\text { conectores y } \\
\text { mecanismos } \\
\text { para recuperar } \\
\text { información } \\
\text { previa están mal } \\
\text { utilizados; presenta } \\
\text { mayoritariamente } \\
\text { ideas solo } \\
\text { yuxtapuestas, } \\
\text { o bien unidas } \\
\text { predominantemente } \\
\text { por relaciones de } \\
\text { adición. }\end{array}$ & $\begin{array}{l}\text { El texto demuestra } \\
\text { una capacidad } \\
\text { básica para utilizar } \\
\text { los recursos de } \\
\text { la coherencia } \\
\text { local. Si bien es } \\
\text { posible que cometa } \\
\text { algunos errores al } \\
\text { hacerlo, se nota } \\
\text { que es capaz de } \\
\text { utilizar relaciones } \\
\text { lógicas, conectores } \\
\text { o mecanismos } \\
\text { para recuperar } \\
\text { información previa } \\
\text { propios de la } \\
\text { oralidad, que no } \\
\text { implican mayor } \\
\text { transformación e } \\
\text { interrelación entre } \\
\text { los contenidos } \\
\text { presentados. }\end{array}$ & $\begin{array}{l}\text { El texto demuestra } \\
\text { una capacidad } \\
\text { emergente para } \\
\text { utilizar los } \\
\text { recursos de la } \\
\text { coherencia local. } \\
\text { Si bien es posible } \\
\text { que cometa algunos } \\
\text { errores, se nota } \\
\text { que es capaz de } \\
\text { utilizar relaciones } \\
\text { lógicas, conectores } \\
\text { o mecanismos } \\
\text { para recuperar } \\
\text { información } \\
\text { previa propios } \\
\text { de la oralidad y } \\
\text { que comienza a } \\
\text { usar recursos más } \\
\text { complejos, propios } \\
\text { de la escritura } \\
\text { expositiva, que } \\
\text { reflejan mayor } \\
\text { transformación e } \\
\text { interrelación entre } \\
\text { los contenidos } \\
\text { presentados. }\end{array}$ & $\begin{array}{l}\text { El texto demuestra } \\
\text { dominio de los } \\
\text { recursos de } \\
\text { la coherencia } \\
\text { local. Utiliza } \\
\text { adecuadamente } \\
\text { una variedad } \\
\text { de relaciones } \\
\text { lógicas, conectores } \\
\text { y mecanismos } \\
\text { para recuperar } \\
\text { información previa } \\
\text { más complejos, } \\
\text { propios de la } \\
\text { escritura, que } \\
\text { reflejan una mayor } \\
\text { transformación e } \\
\text { interrelación entre } \\
\text { los contenidos } \\
\text { presentados. }\end{array}$ \\
\hline
\end{tabular}




\section{Asignación de puntaje}

La asignación de puntaje se realizó con dos correctoras que fueron capacitadas en la aplicación de ambas rúbricas. Para velar por la confiabilidad de los resultados, se realizaron sesiones de $100 \%$ de doble corrección hasta alcanzar niveles satisfactorios de consistencia entre correctoras (un mínimo de 80\%). Una vez obtenida la consistencia, las correctoras puntuaron respuestas sin doble corrección. Primero se puntuaron todas las respuestas con la rúbrica CL y luego con la de RCL. Así también, las correctoras analizaron, para cada rúbrica, primero las respuestas de $5^{\circ} \mathrm{B}$, después las de $1^{\circ} \mathrm{M}$ y finalmente las de $4^{\circ} \mathrm{M}$. Las correctoras asignaron puntajes de 1 a 4 dependiendo del nivel en que se clasificaba la respuesta en cada rúbrica. De este modo, el máximo puntaje que puede obtener una respuesta por rúbrica es de 4 puntos y el mínimo, de 1 punto.

Con el fin de explorar posibles diferencias significativas en los desempeños por nivel escolar y rúbrica, se efectuaron análisis de varianza y, para establecer relaciones entre los desempeños observados en cada una de las dos medidas y en cada nivel escolar, se realizaron cálculos de correlaciones. Todos los análisis estadísticos fueron hechos con el programa SPSS versión 15.

\section{Resultados}

Los resultados de ambos colegios en cada una de las rúbricas (tabla 4) muestran que la medida CL concentra los mejores rendimientos y que obtienen puntajes promedio muy similares ( 2,79 y 2,76, respectivamente), en cambio, se observa que los desempeños de los colegios varían en la medida RCL $(1,58$ y 2,0$)$. Las pruebas ANOVA muestran, consecuentemente, que las diferencias entre ambos establecimientos en la medida CL no son significativas, $F(1,147)=, 241$ p. < 0,624 , pero sí lo son en la medida RCL, $F(1,147)=6,928$ p. $<0,009$.

Tabla 4: Promedios por establecimiento en las dos medidas analizadas

\begin{tabular}{|c|c|c|}
\hline & Establecimiento 1 & Establecimiento 2 \\
\hline \multirow{2}{*}{ CL } & 2,79 & 2,76 \\
& $(0,82)$ & $(0,81)$ \\
\hline \multirow{2}{*}{ RCL } & 1,58 & 2,00 \\
& $(0,80)$ & $(0,92)$ \\
\hline
\end{tabular}

En segundo lugar, se calcularon los desempeños obtenidos por los estudiantes de los tres niveles escolares en cada establecimiento y en las dos dimensiones analizadas. La tabla 5 muestra que los desempeños en RCL son inferiores que los que se obtienen para la medida CL en los tres niveles escolares de ambos colegios. Al comparar entre niveles escolares, se observa que los puntajes promedio tienden a subir gradualmente a lo largo de los tres niveles en RCL, pero no en CL. En relación con esta última medida, en ambos colegios se observa un incremento entre $5^{\circ} \mathrm{B}$ y $1^{\circ} \mathrm{M}$, lo que no sucede entre este último nivel y 
$4^{\circ} \mathrm{M}$. Es decir, se observa una progresión en el desempeño en CL durante el segundo ciclo básico (los años de la preadolescencia, de 10 a 14), pero esta progresión se detiene al comenzar la EM (desde los 14 años). Las pruebas de varianza muestran que las diferencias de desempeño en RCL entre los tres cursos son estadísticamente significativas $F(2,145)=38,887$ p. $<0,000$. Es importante destacar que los cursos de EM de ambos colegios no alcanzan el nivel máximo en ninguna de las dos medidas. En el caso de CL los desempeños de $1^{\circ}$ y $4^{\circ} \mathrm{M}$ se mantienen alrededor del nivel 3 y, en RCL, se sitúan en torno al nivel 2.

Tabla 5: Promedios obtenidos por nivel de escolarización y por medida analizada

\begin{tabular}{|c|c|c|c|c|c|c|}
\hline & \multicolumn{3}{|c|}{ Establecimiento } & \multicolumn{3}{c|}{ Establecimiento 2} \\
\hline & $5^{\circ} \mathrm{B}$ & $1^{\circ} \mathrm{M}$ & $4^{\circ} \mathrm{M}$ & $5^{\circ} \mathrm{B}$ & $1^{\circ} \mathrm{M}$ & $4^{\circ} \mathrm{M}$ \\
\hline $\mathrm{CL}$ & 2,18 & 3,00 & 2,81 & 2,75 & 2,90 & 2,81 \\
\hline $\mathrm{RCL}$ & 1,14 & 2,04 & 2,77 & 1,12 & 1,84 & 2,25 \\
\hline
\end{tabular}

Con el propósito de explorar asociaciones entre los desempeños obtenidos en cada una de las dos medidas, se efectuaron cálculos de correlaciones. Así, se calcularon correlaciones utilizando puntajes promedio obtenidos por toda la muestra en ambas medidas. Los resultados indican que las medidas CL y RCL correlacionan positivamente y de manera estadísticamente significativa $\mathrm{r}(147)=0,197, p<0,01$.

Luego, se efectuaron nuevos cálculos de correlaciones para explorar asociaciones entre los desempeños de las dos medidas, al interior de cada nivel escolar. En $5^{\circ}$ B no se observa una correlación positiva entre ambas medidas, cuestión que sí se advierte para los dos niveles mayores ( $1^{\circ}$ medio y $4^{\circ}$ medio). Es importante destacar que la correlación se incrementa a lo largo de los niveles escolares $\left(5^{\circ} \mathrm{B} \mathrm{r}(71)=-0,84, p<0,01,1^{\circ} \mathrm{M} \mathrm{r}(52)=0,294, p<0,01\right.$ y $4^{\circ} \mathrm{M}$ $r(20)=0,413, p<0,01)$. Es importante indicar que en $4^{\circ}$ medio, el tamaño de la muestra de estudiantes no permite que se reduzca la probabilidad de error estándar, por lo que esta última correlación no aparece estadísticamente significativa, a pesar de que se expresa en una cifra más alta $(, 413)$.

Los ejemplos 1, 2 y 3 muestran desempeños típicos de cada nivel en ambas medidas.

(1) $5^{\circ}$ básico: CL nivel 2 y RCL nivel 1

INSTRUCCIONES
Muchos padres de tu colegio se preocupan por las peleas que hay entre los alumnos.
Escribe un texto para informar a los padres sobre las peleas que hay entre tus
compañeros y lo que se podría hacer para solucionar este problema.


Los problemas que ay en mi colegio son que son muy picados por que abeces cuando estan jugando a la pinta pillan a uno y como al que pillaron no le gusto pillar en piesa a de sir el que lo pillo y so trampa y a y se pica y arma atado y seponen a peliar.

y tambien juegan a las cosas que dan en la tele como la lucha libre y a y en piesan a gugar ala luchas y a veces como le esta ganando en el juego se pica y le pega un combo de verdad y terminan peliando los dos que estan juegando a los juegos de la lucha libre.

Solucion:

Yo qreo que de berian de gar de juegar a los juegos bruscos y si estan peliando ay que separarlos y no degar que pelen y los picotas que no jueguen a los juegos que saben que los ban a pillar y si no les gusta que no jueguen a nada y ce fueran por picotas y yo creo que a los picotas ay que de garlos as ta que a prendan a no cer picota.

El ejemplo ilustra un producto escrito típico del nivel $5^{\circ}$ básico. Dados los problemas de CL que presenta, es necesario hacer frecuentes interrupciones durante la lectura para construir el significado del texto. Así también, es posible observar que las ideas se relacionan predominantemente con el nexo " $y$ " usado con diversas funciones.

\section{(2) $1^{\circ}$ medio: CL nivel 3 y RCL nivel 2}

\section{INSTRUCCIONES}

La violencia en las escuelas del país es un tema que preocupa. Para entender bien este problema, hace falta conocer la versión de los mismos escolares. Escribe un texto en el que describas el problema de la violencia en las escuelas de tu comuna.

Hace más o menos dos años aca ubo una pelea entre dos alumnos tubieron muchos problemas muchas peleas. Ellos por eran muy territoriales todos su problemas terminaron en un ajuste de cuentas pendientes uno de ellos resibio un corte con un tiptop en la cara.

Tambien en otros colegios se esperan distintos bandos a la salida y se pegan con lo que tengan más cerca.

Aquí es muy frecuente ese problema de peleas a la salida de los colegios, donde la fuerza policial tambien a tomado cartas en el asunto.

Ayer 2 de octubre del 2008 ubo un enfrentamiento entre unos compañeros el era peruano y lo discriminaban mucho por eso entonces se aburrio y se tiraron las mesas, las sillas, etc....hasta que se pescaron a combos al medio de la sala y la señorita sin poder hacer nada se puso histerica, al final uno se equinso el dedo y el otro la muñeca y nadie iso nada hasta hoy cuando mandaron a la mitad del curso suspendido.

El ejemplo ilustra un producto escrito típico del nivel $1^{\circ}$ medio. En general es fácil para el lector construir el significado del texto, pues se puede leer fluidamente salvo en un fragmento en que un problema de CL obliga a la relectura. Respecto del dominio de RCL, se observa que las ideas se asocian predominantemente con relaciones lógicas y nexos de adición y temporalidad que las organizan en un mismo nivel, en lugar de servir para interrelacionarlas y transformarlas en nuevas estructuras jerárquicas. 
(3) $4^{\circ}$ medio: CL nivel 3 y RCL nivel 2

\begin{tabular}{|l|}
\hline INSTRUCCIONES \\
Imagina que eres periodista de un diario internacional de internet. Escribe un texto para \\
el diario, en el que informes a personas extranjeras acerca de los problemas actuales \\
de la Educación en Chile. \\
\hline La información en Chile sobre la educación es alarmante ya que la mayoría de los \\
alumnos reclama que la educación deviera ser gratuita ya que en otros paises la \\
educación es gratuita y con mas oportunidades de trabajo. \\
Los alumnos de los establecimientos actúan con protestas ya que es lo unico que \\
pueden haser para llamar la atención del gobierno de educación. \\
Por otro lado lo unico que logran es destrucion en las calles y que algunos alumnos \\
se vallan presos la rebeldía o que ellos sigan adelante para tener una educación mejor \\
y con mas oportunidades a futuro. \\
Se piensa que ellos lo hacen para destruir el pais pero si no los damos cuenta ellos \\
reclaman por algo que le esta pasando siempre el problema de la educacion en chile \\
es un problema que afectan a familias de bajos recursos ya que tienen que postular \\
para ganar vecas pero que pasa con la otra mitad del alumnado que no logra ganar las \\
vecas se quedan sin oportunidades y no les queda de otro que trabajar sin estudiar \\
y de ser algo.
\end{tabular}

El ejemplo ilustra un producto escrito típico del nivel $4^{\circ}$ medio. En general es fácil para el lector construir el significado del texto, pues se puede leer fluidamente salvo en un fragmento en que un problema de CL obliga a la relectura. Respecto del dominio de RCL, se observa que las ideas se asocian predominantemente con relaciones lógicas y nexos de causalidad y adición que las organizan en un mismo nivel, en lugar de servir para interrelacionarlas y transformarlas en nuevas estructuras jerárquicas.

\section{Conclusiones}

Las conclusiones que se presentan a continuación se organizan en torno a dos ámbitos. El primero aborda los resultados considerando fundamentalmente un trabajo previo realizado en Chile que tenía un propósito y una metodología semejantes a los del presente estudio (Concha, 2007) y el otro ámbito intenta interpretar los datos obtenidos desde la perspectiva evolutiva.

En la presente investigación se reitera en EM la asociación entre CL y RCL encontrada previamente (Concha 2007), reflejando que a mayor dominio de los recursos más propios del discurso escrito expositivo se incrementa también la coherencia de su escritura. Sin embargo, en $5^{\circ}$ básico la correlación comentada no se observa, ampliando la evidencia del estudio previamente mencionado. El no encontrar correlación en $5^{\circ}$ básico podría deberse al bajo dominio que estos alumnos poseen de los RCL, lo que se manifiesta en que sus discursos se caracterizan por expresar ideas asociadas mediante la adyacencia o a través 
del conector "y" que adquiere múltiples funciones. En consecuencia, los RCL no aportarían a la construcción coherente de discursos escritos, por lo que el desempeño alcanzado en coherencia en este nivel escolar se explicaría por una transferencia directa del dominio que los estudiantes poseen de la oralidad y no por la utilización de los recursos propios del lenguaje escrito.

Un resultado interesante con respecto a los estudiantes de EM es que aun cuando ellos incrementan significativamente el dominio de los señalados recursos a través de los niveles escolares, este aporte no sería suficiente como para aumentar de forma determinante el puntaje promedio de CL en $1^{\circ}$ y $4^{\circ}$ medio, el que se mantiene en torno al nivel 3. En efecto, la mayoría de los sujetos de EM que participaron en este trabajo se ubicaron en torno al nivel 2 de la rúbrica RCL, lo que significa que sus productos escritos no reflejan un razonamiento respecto de cómo se relacionan las distintas ideas que incluyen (Durst, 1987). Priman aún en sus textos recursos que no son característicos de la escritura expositiva, como es el caso de la adición y la temporalidad. Además, las ideas se presentan en un mismo nivel en lugar de haber sido transformadas e interrelacionadas en nuevas estructuras de información, como es propio de la producción escrita académica (Bereiter y Scardamalia, 1987). Así, para la mayoría de los sujetos, su dominio de RCL no parece ser suficiente como para aumentar de modo sustancial la CL, mientras que, como revelan los cálculos de correlaciones, aquellos que presentaron más dominio de los recursos lingüísticos convencionales de la escritura expositiva, producen textos que son percibidos de manera más coherente por los lectores.

Con respecto a los puntajes de la CL, se advierte que en los tres cursos se incrementan, sin embargo, no se produce un aumento estadísticamente significativo. De este modo, los alumnos de los tres cursos obtienen puntajes promedios similares (entre el nivel 2 y el 3). Ello significa que los alumnos de los niveles escolares estudiados producen textos en los que existen algunas secuencias incoherentes que no impiden al lector determinar el sentido global del escrito. No obstante, llama la atención que no se alcance un dominio total de la coherencia local ni en $1^{\circ}$ ni en $4^{\circ}$ medio, cuestión que se opone a datos obtenidos en investigaciones internacionales que indican que entre los 14 y los 16 años los sujetos son capaces de producir discursos coherentes (Scardamalia y Paris, 1985, Langer, 1986). Una posible interpretación es suponer que tempranamente se adquiere la capacidad para comunicarse coherentemente mediante recursos de la oralidad y que los sujetos de EM estudiados mantienen este recurso como la estrategia principal para obtener la coherencia. Esto sugiere que la experiencia escolar podría no estar siendo suficientemente efectiva en entregar herramientas que permitan que los estudiantes utilicen los recursos característicos del discurso académico para construir la CL de los textos que escriben.

El mejor desempeño en CL también se observó al comparar los rendimientos de los dos colegios ya que se determinó que se comportaban de manera similar en esta medida, lo que es consistente con su homogeneidad en cuanto a tipo de dependencia y rendimiento SIMCE. No obstante, sus desempeños fueron

188


diferentes en la medida RCL, lo que podría interpretarse como que dichos recursos son más sensibles al efecto de las prácticas pedagógicas y al entrenamiento específico que hayan tenido los estudiantes con ellos. Esta interpretación puede sustentarse en los resultados observados por Concha (2007) respecto de que la mayoría de los estudiantes de $4^{\circ}$ medio que mostraban mejor dominio de RCL, informaron haber recibido instrucción directa sobre el significado y función de los conectores lógicos en su experiencia escolar, mientras que gran parte de los estudiantes con bajo dominio de RCL, no recordaba este tipo de experiencia. También la sugerencia de Lee (2002) de que la Coherencia Local no solo se desarrolla sino que es necesario enseñarla apoya la evidencia expuesta.

Con relación a la evolución de ambas medidas, se advierte que los resultados son distintos para CL y RCL. En el caso de RCL, existió un incremento estadísticamente significativo en los puntajes a lo largo de los tres niveles escolares, lo que permite hipotetizar un desarrollo del dominio de los recursos de la lengua (conectores, relaciones lógicas y mecanismos para recuperar información) involucrados en la generación de coherencia local, en el tipo de texto expositivo. Lo anterior replica estudios previos con poblaciones angloparlantes que señalan que el dominio de RCL como conectores y relaciones lógicas es uno de los aspectos que más visiblemente evoluciona a lo largo de la EB en el ámbito del desarrollo de la escritura no narrativa (Langer, 1986; Mc Cutchen y Perfetti, 1983). Los resultados de este trabajo plantean que dichos recursos continúan su evolución a lo largo de la EM, no obstante los estudiantes de $1^{\circ}$ medio y $4^{\circ}$ medio de la muestra no alcanzan un total dominio de este desempeño. En sus productos escritos aún se observa que construyen la coherencia mediante mecanismos más propios de la oralidad. Lo anterior contrasta con investigaciones extranjeras puesto que problematizan la postura de que en EM se haya alcanzado un nivel satisfactorio de los señalados recursos (Langer, 1986; Piolat, Roussey y Gombert,1999).

Respecto de la CL, los resultados indican que es mayor el incremento que se produce entre $5^{\circ}$ básico y $1^{\circ}$ medio que entre los dos cursos de EM, no obstante en ninguno de los tramos existe un aumento estadísticamente significativo. Estos datos podrían estar mostrando que muy tempranamente se adquiere la capacidad de producir un texto que, si bien presenta algunas secuencias incoherentes, permite al lector construir la coherencia global. Esta capacidad, sin embargo, no mejora sustancialmente a lo largo de la escolaridad, de tal manera que los estudiantes de EM presentan un comportamiento muy similar a los de EB. Al respecto es importante reiterar lo sugerido por los trabajos anteriormente descritos (Concha 2007; Lee, 2002) en que se enfatiza que es posible mejorar el desempeño en coherencia por la vía de la educación explícita, en especial, a través de la instrucción en los recursos de la coherencia local.

En futuras investigaciones sería importante ahondar en el tipo de didáctica específica que pudiera estar asociada con un mejor dominio de estos recursos; así también, sería relevante explorar qué tipo de metodologías didácticas se utilizan en las aulas del país para desarrollarlos. Como señalara Silvestri (2002), 
la adquisición discursivo-textual que implica la habilidad para comprender y producir textos -orales o escritos- de diversos tipos y géneros depende de las oportunidades de interacción cultural que tengan los sujetos. Desde esta perspectiva, los resultados aquí expuestos podrían estar develando una carencia de los estudiantes en cuanto a experiencias de interacción con los discursos académicos, que harían de toda relevancia emprender un trabajo de didáctica específica para remediar dichas carencias.

\section{Bibliografía}

Bereiter, C. \& Scardamalia, M. (1987).

The psychology of written composition. Hillsdale, New Jersey: Laurence Erlbaum Associates.

Berman, R. \& Nir-Sagiv, T. (2007).

"Comparing Narrative and Expository Text Construction Across Adolescence. A Developmental Paradox". Discourse Processes, 43(2), 79-120.

Borzone, A. Y Diuk, B. (2003).

"La escritura de textos en niños pequeños: conocimiento ortográfico y producción textual". Cultura y Educación, 15, 1, $17-27(11)$.

Carlino, P. (2008). "Prácticas y representaciones de la escritura en la Universidad". Actas del I Congreso de Nacional de Estudios Comparados en Educación, Buenos Aires, 18 de noviembre de 2005.

Charolles, M. (1983). "Towards a heuristic approach to text-coherence problems". In Nebauer, F. (Ed.) Coherence in natural-language texts. Hamburg: Buske, Helmut Buske Verlag Hamburg.

Clark, E. (2003). First language acquisition. Cambridge, UK: Cambridge University Press.

Crespo, N. (1998). "La evaluación de la escritura: posibles criterios". Signos, 31(43-44), 153- 164.

Concha, S. (2007). L Local coherence in academic writing: An exploration of 12th grade Chilean Spanish monolingual students, metalinguistic knowledge, writing process and writing products. Tesis doctoral no publicada, Boston University.

Durst, R. (1987) "Cognitive and linguistic demands of academic writing". In Langer, J. and Applebee, A. (Eds.) Research in the Teaching of English, Vol. 21, No. 4, 347-375.

Favart, M. \& Passerault, J.M. (1996) "Functionality of cohesion devices in the management of local and global coherence: Two studies in children's written production of narratives". In Rijlaarsdam, G., van den Bergh, H. \& Couzijn, M. (Eds.) Theories, models 
Hayes, J.R (2000). "A new framework for understanding cognition and affect in writing". In Indrisano, R. \& Squire, J.R (Eds.) Perspectives on Writing. Newark, DE: International Reading Association.

Hobbs, J.R. (1983). "Why is discourse coherent?" In Nebauer, F. (Ed.) Coherence in Natural language Texts. Hamburg: Buske, Helmut Buske Verlag Hamburg.

Jacobs, S. (1990) "Building Hierarchy: Learning the language of the science domain, ages 10-13" (p. 151-169). In Connors U., Johns, A. (Eds.) Coherence in writing. Alexandria, Virginia: Library of Congress Catalog.

Kellog, R. (1987) "Effects of topic knowledge on the allocation of processing time and cognitive effort to writing processes". Memory and Cognition, Vol. 15, No. 3, 256-266.

Langer, J. (1986). Children reading and writing. Structures and Strategies. New Jersey: Abbley.

Lee, I. (2002) "Teaching coherence to ESL students: a classroom inquiry". Journal of Second Language Writing, 11, 135-159.

Marinkovich, J. y Poblete, C. (2000).

Un intento de evaluar el conocimiento acerca de la escritura en estudiantes de enseñanza básica. Signos, 33(47), 101-110.

McCutchen, D., \& Perfetti, C. (1983).

"Local coherence: Helping young writers manage a complex task". The Elementary School Journal, The University of Chicago, Vol. 84, No. 1, 71-75.

Parodi, G. (2000). "La evaluación de la producción de textos escritos argumentativos: una alternativa cognitivo / discursiva". Signos, 33(47), 151-166.

Peterson, C. \& McCabe, A. (1991).

"Linking children's connective use and narrative macrostructure". In Peterson, C. \& McCabe, A. (Eds.) Developing narrative structure. Hillsdale, NJ: Lawrence Erlbaum.

Piolat, A., Roussey, J. \& Gombert, A. (1999)

"The development of argumentative schema in writing". En Andriessen, J. y Coirier, P. (Eds) Foundations of Argumentative Text Processing. Amsterdam: Amsterdam University Press.

Scardamalia, M. \& Paris, P. (1985).

"The Function of Explicit Discourse Knowledge in the Development of Text Representations and Composing Strategies". Cognition and Instruction 2(1), 1-39.

Silvestri, A. (2002). "Funciones psicológicas y adquisición discursiva". Propuesta educativa, 25. 
Escritura expositiva en tres niveles de escolaridad: / Dra. Concha; Mg. Aravena, Mg. Coloma y Mg. Romero

Velásquez, M. y Alonso, T. (2007).

"Desarrollo y transferencia de estrategias de producción escrita". Signos, 40(63), 219-238.

Wikborg, E. (1990). "Types of coherence breaks in Swedish student writing: Misleading paragraph division". In Connor, U., Johns, A.M (Eds.). Coherence in Writing: Research and Pedagogical Perspectives. Alexandria, Virginia: Teachers of English to Speakers of Other Languages. 